\title{
High energy neutrino production by cosmic ray interactions in the Sun
}

\author{
G. Ingelman* \\ Department of Radiation Sciences, Uppsala University, Box 535, S-751 21 Uppsala, Sweden \\ and Deutsches Elektronen-Synchrotron DESY, Notkestrasse 85, D-22603 Hamburg, Germany \\ M. Thunman ${ }^{\dagger}$ \\ Department of Radiation Sciences, Uppsala University, Box 535, S-751 21 Uppsala, Sweden
}

(Received 12 April 1996)

\begin{abstract}
The flux of neutrinos originating from cosmic ray interactions with matter in the Sun has been calculated based on Monte Carlo models for high energy particle interactions. The resulting flux at the Earth (within the Sun's solid angle) is higher than the corresponding one from cosmic ray interactions with Earth's atmosphere. The smallness of the absolute rate, however, precludes it as a practical "standard candle" for neutrino telescopes and limits neutrino oscillation searches. On the other hand, it facilitates dark matter searches based on neutrinos from neutralino annihilation in the Sun. [S0556-2821(96)03019-6]
\end{abstract}

PACS number(s): 96.40.Tv, 13.60.Hb, 26.65.+t, 96.40.De

\section{INTRODUCTION}

High energy cosmic ray particles, mainly nucleons, interact with matter to produce secondary particles that are mainly mesons and some baryons. This applies in particular to cosmic rays entering the atmosphere of the Sun. The produced particles propagate through the Sun until they either decay or make secondary interactions producing new particles that contribute further to develop a cascade. Decays of particles in such cascades will produce neutrinos and other leptons, e.g., muons that in turn decay into neutrinos. This scenario is similar to the cascades induced by cosmic rays in Earth's atmosphere, which we have studied extensively [1]. However, the solar atmosphere is less dense at the typical interaction heights and, therefore, a larger fraction of the mesons will decay instead of interacting. This leads to relatively more neutrinos produced in the Sun as compared to the Earth.

It has been proposed [2] that the Sun might be used as a "standard candle" for neutrino telescopes, which is only possible if the flux is significantly higher than Earth's atmospheric flux. A large such neutrino flux from the Sun would, on the other hand, be a severe background for searches of neutrinos from neutralino annihilation in the Sun [3]. The hypothetical neutralinos appear in theories based on supersymmetry (SUSY) [4] and are of fundamental interest in particle physics as well as in cosmology since they could contribute to the dark matter in the Universe.

In this paper we study the production of muon and electron neutrinos in cosmic ray interactions in the Sun, as well as their propagation through the Sun and to the Earth where they could be detected in neutrino telescopes, such as the Antarctic Muon and Neutrino Detector Array (AMANDA) [5], BAIKAL [6], Deep Underground Muon and Neutrino Detector (DUMAND) [7], and NESTOR [8]. The cascade interactions in the Sun are treated in detail using Monte Carlo methods to

\footnotetext{
*Electronic address: ingelman@tsl.uu.se

†Electronic address: thunman@tsl.uu.se
}

simulate the high energy particle interactions. In particular, the Lund model [9] and Monte Carlo programs [10] are invoked. The resulting neutrino fluxes at the Earth are compared with the fluxes from cosmic ray interactions in Earth's atmosphere [1]. This solar neutrino flux is discussed in terms of the above "standard candle" idea and neutralino search. We also investigate the possibility of neutrino oscillations taking place between the source at the Sun and the detector at the Earth.

\section{MODELS AND CALCULATIONAL TECHNIQUES}

\section{A. Cosmic ray spectrum}

The flux of primary cosmic ray particles is conventionally parametrized as $[1,11,12]$

$$
\phi_{N}(E)\left[\frac{\text { nucleons }}{\mathrm{cm}^{2} \mathrm{~s} \mathrm{sr} \mathrm{GeV} / A}\right]=\left\{\begin{array}{cc}
1.7 E^{-2.7} & E<5 \times 10^{6} \mathrm{GeV}, \\
174 E^{-3} & E>5 \times 10^{6} \mathrm{GeV} .
\end{array}\right.
$$

The normalization is derived here [13] from the directly measured primary spectrum using balloon-borne emulsion chambers in JACEE [14]. It agrees (within some 10\%) with more indirectly derived spectra based on measured atmospheric muon fluxes [15], and is also compatible with the data discussed in [16]. For the energies of our interest $(E \gtrsim 100 \mathrm{GeV})$, we take the cosmic ray flux to be isotropic, since the anisotropy is $\$ 5 \%$ [17].

The cosmic ray composition is dominated by protons with only a smaller component of nuclei $[13,16]$. Likewise, the outer parts of the solar atmosphere consist mainly of hydrogen with only a small fraction of helium. Therefore, the interactions producing the secondary particle fluxes are dominantly proton-proton collisions. The small contribution of nuclear collisions can also be treated as nucleon-nucleon interactions, since the nuclear binding energies are negligible and other nuclear effects have little influence on the high energy secondary particles which we are interested in. 
TABLE I. Parameters for the solar density profile in Eq. (2).

\begin{tabular}{lcc}
\hline \hline Height $h(\mathrm{~km})$ & $\rho_{0}\left(\mathrm{~g} / \mathrm{cm}^{3}\right)$ & $h_{0}(\mathrm{~km})$ \\
\hline$h>0$ & $3.68 \times 10^{-7}$ & 115 \\
$-2000<h<0$ & $3.68 \times 10^{-7}$ & 622 \\
$h<-2000$ & $45.3 \times 10^{-7}$ & 2835 \\
\hline \hline
\end{tabular}

\section{B. Solar matter distribution}

The interaction of cosmic ray particles in the Sun can be treated analogously to our calculation of cosmic ray interactions in the atmosphere of the Earth [1]. However, the atmosphere of the Sun is less dense and particles may therefore propagate deeper in to the Sun such that a more elaborate matter density profile is needed. Although there is no welldefined solar surface, it is convenient to consider different regions in relation to the solar radius $R_{\odot}=6.96 \times 10^{5} \mathrm{~km}$. One may then apply an exponential density profile

$$
\rho(h)=\rho_{0} e^{-h / h_{0}},
$$

where $h>0$ and $h<0$ correspond to locations above and below $R_{\odot}$, respectively. The parameters $\rho_{0}$ and $h_{0}$, given in Table I, were obtained by fitting the data of [18] for the atmosphere and of [19] for the interior of the Sun. For muons which can reach deep into the Sun $(\sim 20000 \mathrm{~km}$ vertical depth), a third region is introduced to get an adequate description of the density profile.

To calculate the flux of particles from the Sun that reach the Earth one must also consider where in the Sun the primary cosmic ray particle interacts. The essential point is whether the secondary particles and final neutrinos only have to pass through the solar atmosphere or through the higher density interior before reaching the Earth. This corresponds to an impact parameter $b$ that varies between $R_{\odot}$ (peripheral hit) and zero (central hit) and a correspondingly varying effective density function along the particle trajectories. An exact treatment of this in our Monte Carlo simulation becomes quite complex. We have therefore simplified the situation by making the simulations for fixed impact parameter values $\left[b=0, b=2 R_{\odot} / 3\right.$ (geometric average) and $\left.b=R_{\odot}\right]$ and then interpolated between them for the integrated results taking neutrino attenuation into account.

\section{Basic particle interactions}

The interaction of the cosmic ray particles with the solar material is treated as basic proton-proton collisions at high center-of-mass system (c.m.s.) energy. The production of secondary particles and their decay into neutrinos can then be simulated in great detail using the Lund Monte Carlo programs PYTHIA and JETSET [10]. The neutrino flux arises from the decay of ordinary mesons, mainly $\pi$ and $K$, and from decay of muons.

The production of ordinary hadrons (not containing heavy quarks) is dominantly through minimum bias hadron-hadron collisions. The strong interaction mechanism is here of a soft nonperturbative nature that cannot be calculated based on proper theory, but must be modelled. In the successful Lund model [9] hadron production arises through the fragmentation of color string fields between partons scattered in semi- soft QCD interactions [10]. The essentially one-dimensional color field arising between separated color charges is described by a one-dimensional flux tube whose dynamics is taken as that of a massless relativistic string. Quarkantiquark pairs are produced from the energy in the field through a quantum mechanical tunneling process. The string is thereby broken into smaller pieces with these new color charges as end points and, as the process is iterated, hadrons are formed. These obtain limited momenta transverse to the string (given by a Gaussian of a few hundred $\mathrm{MeV}$ width) but their longitudinal momentum may be large as it is given by a probability function in the fraction of the available energy momentum in the string system taken by the hadron. All mesons and baryons in the basic multiplets may be produced and the subsequent decays are fully included. The iterative and stochastic nature of the process is the basis for the implementation of the model in the JETSET program [10].

A non-negligible contribution to the inclusive cross section is given by diffractive interactions. These are also modeled in PYTHIA [10] using cross sections from a wellfunctioning Regge-based approach and simulating the diffractively produced final state using an adaptation of the Lund string model. These diffractive events are included in our simulations and contribute rather less than $10 \%$ to the final results.

In our study of the neutrino flux from cosmic ray interaction in the Earth's atmosphere [1], an important point was the production of charmed particles. Their prompt decays give a non-negligible contribution to the neutrino flux at very high energies $\left(E_{\nu} \gtrsim 10^{6} \mathrm{GeV}\right)$. This is due to the increase in the $\pi$ and $K$ decay lengths with increasing energy, such that the probability for interacting before they decay increase. The lower density in the solar atmosphere implies that their interaction length is larger, such that they still dominantly decay rather than being lost through interactions. Therefore, so-called prompt neutrinos from charm decay are not as important as for the lepton fluxes in Earth's atmosphere.

\section{Cascade evolution}

To describe the evolution of a cascade in the solar medium we use the same formalism as for interactions in Earth's atmosphere and therefore refer to our earlier study in [1] for more details.

The flux of nucleons in the solar atmosphere develops according to the cascade equation

$$
\frac{d \phi_{N}}{d X}=-\frac{\phi_{N}}{\lambda_{N}}+S(N A \rightarrow N Y),
$$

where $X$ is the depth in the atmosphere and

$$
\lambda_{N}(E)=\frac{\rho(h)}{\Sigma_{A} \sigma_{N A}(E) n_{A}(h)},
$$

is the nucleon interaction length in terms of the number density, $n_{A}(h)$, of nuclei $A$ at height $h$ and the nucleon-nucleus inelastic cross section, $\sigma_{N A}(E)$. The first term in Eq. (3) is the loss of nucleons due to interactions and $S(N A \rightarrow N Y)$ is the regeneration due to interactions of primary nucleons with higher energies. 
This transport equation is solved through a cascade simulation algorithm as follows. A cosmic ray proton is generated with energy drawn from a flat distribution in $\log E$, and a weight assigned to reproduce the shape of the primary spectrum Eq. (1). This primary proton is then propagated down through the solar medium according to Eq. (3) without the regeneration term $S(N A \rightarrow N Y)$ resulting in the solution

$$
\phi(h)=\phi_{0} e^{-X / \lambda_{N}}
$$

From this one can obtain the height of the primary interaction by solving the equation

$$
-\ln \frac{\phi(h)}{\phi_{0}}=-\ln R=\int_{\infty}^{h} \operatorname{dh\sigma n}(h)
$$

for $h$, where $R \in] 0,1[$ is a uniformly distributed random number.

A proton-proton interaction is then generated in full detail with PYTHIA [10] resulting in a complete final state of particles. Secondary particles are followed through the atmosphere where they decay or interact producing cascades. Secondary nucleons give a flux that is rather small compared to the primary flux and could therefore be neglected as a first approximation. We do, however, include the main part of this effect by taking into account secondary nucleons that have an energy of at least $30 \%$ of the primary one. Nucleons with a lower energy give a negligible contribution compared to the primary flux due to its steep energy spectrum Eq.,(1). These leading nucleons emerging in the interactions are therefore allowed to generate a secondary interaction at a height calculated according to

$$
-\ln \frac{\phi(h)}{\phi(H)}=-\ln R^{\prime}=\int_{H}^{h} d h \sigma n(h),
$$

where $H$ is the production height of the nucleon. The procedure is iterated until the energy of the leading nucleon from an interaction falls below $30 \%$ of the primary cosmic ray proton energy.

The secondary mesons are propagated down into the Sun until they either decay or interact, which is decided by comparing simulated interaction and decay lengths. The interaction length is calculated analogous to Eq. (7), while the decay length is given by

$$
L_{\mathrm{dec}}=-c \beta \gamma \tau \ln R \text {. }
$$

Particle decays are fully simulated with daughter particle momenta. In the case of interactions, the interacting particle is regenerated in the same direction but with degraded energy, chosen according to the appropriate leading particle spectrum. Considering only the most energetic "leading" particles in secondary interactions is justified because they give the dominant contribution to the high energy neutrino flux. Other particles with lower energy are much fewer than the particles of the same type and energy produced in primary interactions. Moreover, secondary interactions take place deeper in the atmosphere, where the density is higher, giving a higher probability that a produced meson interacts rather than decays. This further decreases the importance of particles from secondary interactions.
The particle-decay-interaction chain is then repeated until all particles have decayed or their energy fallen below a minimum of $100 \mathrm{GeV}$. The energy spectra for neutrinos are finally obtained by simply counting the number of generated neutrinos and applying the weight assigned to the primary proton. The contributions from decays of the different mesons are shown in Fig. 1 for the different impact parameters $b$. The contribution from charmed and heavier mesons is not included, but are unimportant as will be discussed in Sec. III.

\section{E. Muon propagation in the Sun}

Since muons do not feel the strong force their propagation through matter is quite different from the hadrons. Muons interact electromagnetically and rarely experience hard interactions with a single large energy loss. Instead they typically lose small amounts of energy in each collision, which can be added such that the energy loss can be treated as a continuous process and parameterized in the form [20]

$$
\frac{d E}{-d h}=-\alpha \rho-\beta \rho E,
$$

with $\alpha=0.0025 \mathrm{GeV} /\left(\mathrm{g} / \mathrm{cm}^{2}\right)$ and $\beta=4.0 \times 10^{-6}(\mathrm{~g} /$ $\left.\mathrm{cm}^{2}\right)^{-1}$. This continuous energy loss makes the above treatment of decay versus interaction based on decay and interaction lengths unsuitable. Instead, we apply a method where small steps in energy loss $(\Delta E / E \simeq 10 \%)$ are taken, under which the energy loss rate is approximately constant (i.e., $d E / d h \simeq$ const). The decay probability in such a step can then be calculated analytically and Monte Carlo simulated. If a muon survives such a step in energy the procedure is repeated until the muon decays or gets an energy lower than our $100 \mathrm{GeV}$ cut off.

The resulting neutrino flux from muon decays are shown in Fig. 1 for the three different impact parameters. For muon production with impact parameter $b=R_{\odot}$ the effective matter thickness is very small resulting in no significant energy loss, such that the muons effectively propagate without interactions.

\section{F. Neutrino flux attenuation in the Sun}

The neutrino flux will be attenuated through weak interactions with the nucleons of the solar medium. In charged current interactions the neutrino is lost altogether and instead the corresponding charged lepton emerges, which loses significant energy or is absorbed since this process mainly occurs in the dense interior of the Sun. In a neutral current interaction the neutrino emerges with a reduced energy. We treat this using the analytic technique developed for atmospheric cascades of hadrons [1] and express the flux by

$$
\frac{d \phi_{\nu}}{d X}=-\frac{\phi_{\nu}}{\lambda_{\nu}}+S(\nu A \rightarrow \nu Y) .
$$

The first term, the absorption term, gives the loss due to both charged and neutral current interactions. The second gives the regeneration due to neutral current interactions of neutrinos of higher energies. The latter term is given by 

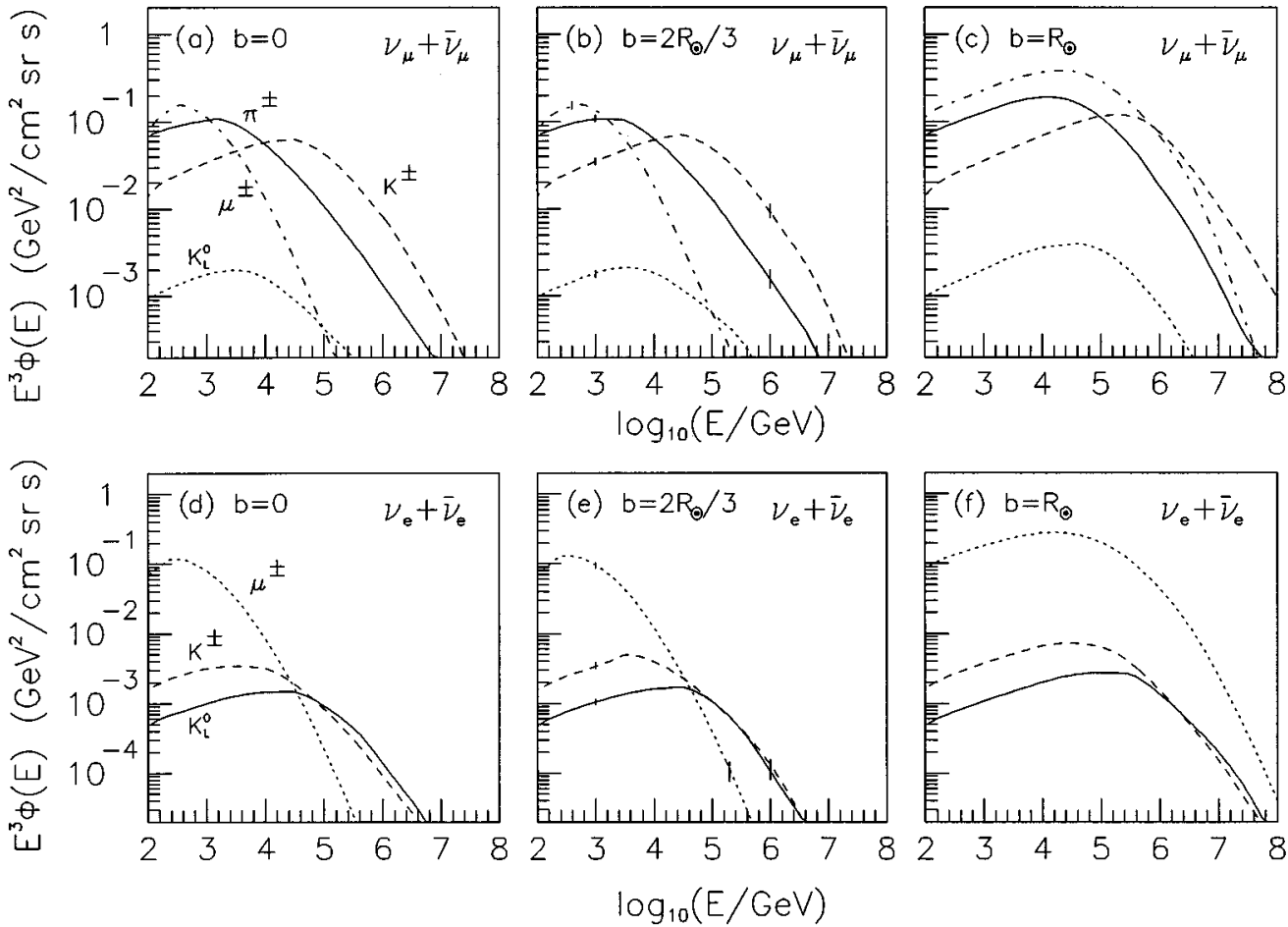

FIG. 1. The $E^{3}$-weighted flux of muon-neutrinos $\left(\nu_{\mu}+\bar{\nu}_{\mu}\right)$ and electron-neutrinos $\left(\nu_{e}+\bar{\nu}_{e}\right)$ from decays of the specified particles $(\pi, K, \mu)$ produced in cascade interactions in the Sun at different impact parameters $b$. The error bars in (b) and (e) indicate the statistical precision (bin size 0.1 in $\log _{10} E$ ) of the Monte Carlo simulation. Note, the neutrino flux attenuation from propagation through the Sun (Sec. II F) is not included.

$$
S(\nu A \rightarrow \nu Y)=\int_{E}^{\infty} d E^{\prime} \frac{\phi_{\nu}\left(E^{\prime}\right)}{\lambda_{\nu}\left(E^{\prime}\right)} \frac{d n_{\nu A \rightarrow \nu Y}\left(E^{\prime}, E\right)}{d E}
$$

and can be rewritten as

$$
\begin{aligned}
S(\nu A & \rightarrow \nu Y) \\
& =\frac{\phi_{\nu}(E)}{\lambda_{\nu}(E)} \int_{E}^{\infty} d E^{\prime} \frac{\phi_{\nu}\left(E^{\prime}\right)}{\phi_{\nu}(E)} \frac{\lambda_{\nu}(E)}{\lambda_{\nu}\left(E^{\prime}\right)} \frac{d n_{\nu A \rightarrow \nu Y}\left(E^{\prime}, E\right)}{d E} \\
& =\frac{\phi_{\nu}(E)}{\lambda_{\nu}(E)} Z,
\end{aligned}
$$

where the integral defines the regeneration $Z$ moment [1]. To use this formula it is normally assumed that the dependence on the depth $X$ cancels in the ratio $\phi_{\nu}\left(E^{\prime}\right) / \phi_{\nu}(E)$, which it does if the interaction length is only weakly energy dependent. Although this is not the case here, this formula is still applicable since the $d n / d E$ spectra are peaked at low energy loss (for high energies). Furthermore, one can use the same $Z$ moment for all neutrinos $\left(\nu_{e}, \bar{\nu}_{e}, \nu_{\mu}, \bar{\nu}_{\mu}\right)$ since their spectral form before attenuation in the Sun are approximately the same and their cross sections are approximately the same. The $Z$ moment is calculated with PYTHIA [10] using a standard technique [1] and it is shown in Fig. 2. Now, Eq. (10) can be solved to give the attenuated neutrino flux

$$
\phi_{\nu}(r)=\phi_{\nu, 0} \exp \left\{-(1-Z) X(r) / \lambda_{\nu}\left(E_{\nu}\right)\right\}
$$

where $X(r)$ is the amount of material being traversed.
The above treatment is of course a simplification. For example, the flux, $\phi(E)$ is a function of the impact parameter $b$. Here, a fit to the flux integrated over the Sun is used. Compared to the two most rough simplifications that can be done, neglecting secondary neutrinos and neglecting energy loss in neutral current interactions, our simplifications are reasonable. By comparing the $Z$ moments, which are zero if secondary neutrinos are neglected and $\sigma^{\mathrm{NC}} / \sigma^{\mathrm{NC}+\mathrm{CC}} \sim 2 / 7$ if energy loss is neglected in neutral current interactions, we can see that the above treatment is a significant improvement.

In principle, each neutrino should be followed through the Sun with the attenuation folded in to get the final attenuated spectrum. However, since the neutrino production only occurs in a tiny outer fraction of the Sun one may use a simpler factorixed approximation. The previously obtained fluxes

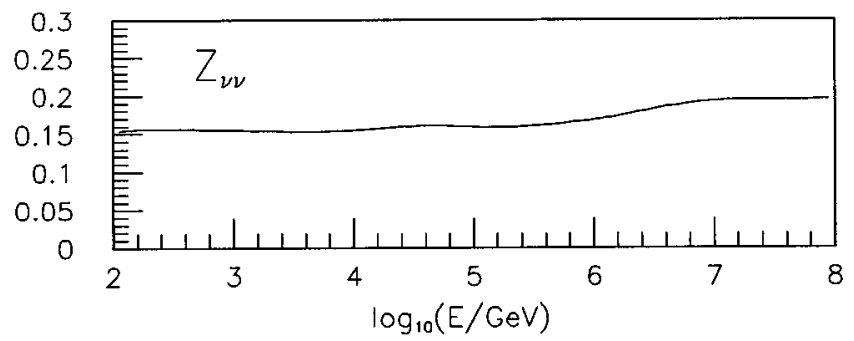

FIG. 2. Energy dependence of the regeneration $Z$ moment, Eq. (12), for neutrino-nucleon interactions. 


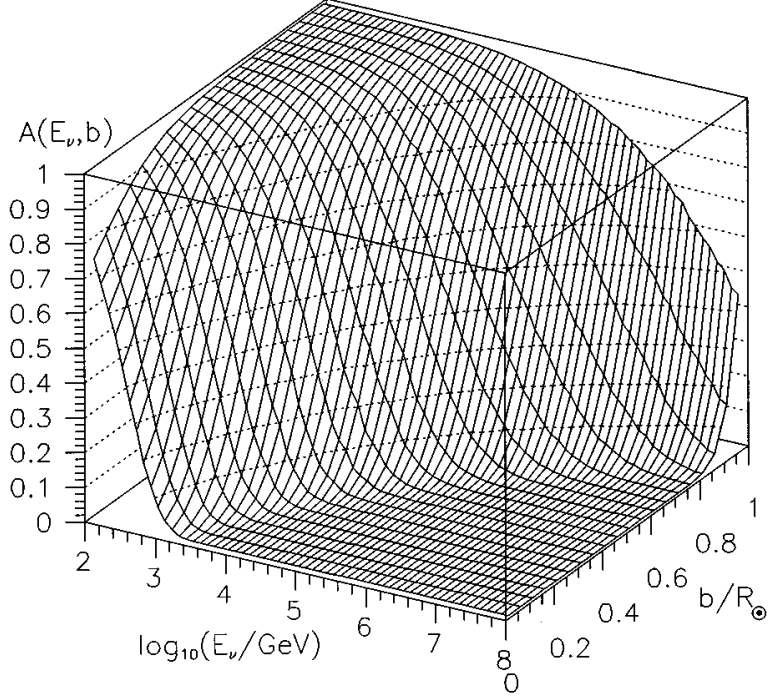

FIG. 3. The attenuation factor, Eq. (14), for neutrinos passing through the Sun as a function of neutrino energy and impact parameter $b$.

can thus be multiplied by an overall attenuation function for the Sun. This attenuation function can then be calculated in terms of the impact parameter

$$
A\left(E_{\nu}, b\right)=\exp \left\{-\sigma\left(E_{\nu}\right)(1-Z) X(b) / m_{N}\right\},
$$

where $X(b)$ is the effective thickness of the Sun and $m_{N}$ is the nucleon mass. A numerical evaluation of this function is shown in Fig. 3.

\section{RESULTING NEUTRINO FLUXES}

The unattenuated neutrino flux from the decay of different particles produced in cosmic ray collisions in the Sun are shown in Fig. 1. As can be seen from the results for different impact parameter values, secondary interactions of mesons and energy loss for the muons are important and give substantially less high energy neutrinos at more central interactions. By interpolating over different impact parameters and folding with the attenuation factor, Eq. (14), the integral over the solar disc is carried out resulting in the total neutrino fluxes at the Earth shown (by solid curves) in Fig. 4. The fluxes are compared with the horizontal [21], the vertical [1], and the prompt [1] fluxes from cosmic ray interactions in Earth's atmosphere.

As demonstrated in Fig. 4, the fluxes from the Sun are significantly higher than those from the Earth's atmosphere. The lower density of the solar atmosphere gives a lower probability for secondary interactions and hence favors decays into high energy neutrinos. For the muon neutrinos, the solar flux is thus about 1 (2) orders of magnitude larger than the horizontal (vertical) atmospheric flux in the energy range $10^{4}-10^{7} \mathrm{GeV}$. In the case of electron neutrinos, the solar flux is very much higher at all energies. This is due to the contribution from muon decays (see Fig. 1), which is only important for the very lowest energies in the atmospheric fluxes. For both muon and electron neutrinos, the slope at high energies $\left(\gtrsim 10^{6} \mathrm{GeV}\right)$ is steeper for the solar fluxes compared to atmospheric ones. This is because of neutrino attenuation, Eq. (14) and Fig. 3, is here getting noticeable in the Sun.

As mentioned earlier the prompt neutrino contribution, i.e., from hadrons with charm and heavier quarks, has not been explicitly studied. The prompt atmospheric fluxes [1] are plotted in Fig. 4 (curve $P$ ) for comparison. Due to the short charmed particle lifetime, these fluxes only depend on the production rate up to $\sim 10^{7} \mathrm{GeV}$. The production in the Sun would therefore be the same, but the attenuation in the Sun results in a prompt neutrino flux that is lower than the atmospheric one.

Our solar muon neutrino flux is, in Fig. 4, compared with

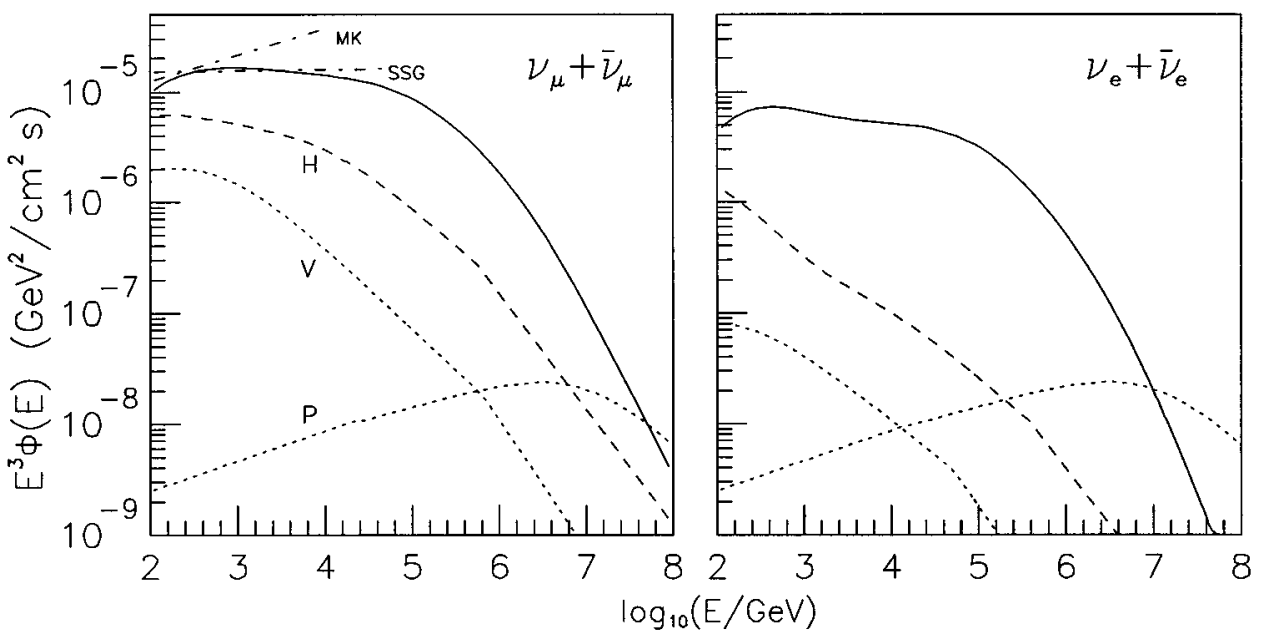

FIG. 4. Cosmic ray induced $E^{3}$-weighted neutrino fluxes at the Earth integrated over the solid angle of the Sun. The fluxes from the Sun obtained in this study (solid lines) are compared with the earlier calculation SSG [22] and the one MK derived from [2], as well as those from Earth's atmosphere as calculated for the vertical flux (curve $V$ ) [1], the horizontal flux (curve $H$ ) [21], and the prompt charm-induced flux (curve $P$ ) [1]. 
TABLE II. Values of parameters in Eq. (15) obtained from fits to the attenuated neutrino fluxes given by the solid lines in Fig. 4.

\begin{tabular}{ccccccc}
\hline \hline & $N_{0}$ & $\gamma$ & $A$ & $E_{0}$ & $\gamma^{\prime}$ & $N_{0}^{\prime}$ \\
\hline$\nu_{\mu}+\bar{\nu}_{\mu}$ & $1.3 \times 10^{-5}$ & 1.98 & $8.5 \times 10^{-6}$ & $3.0 \times 10^{6}$ & 2.38 & $5.1 \times 10^{-3}$ \\
$\nu_{e}+\bar{\nu}_{e}$ & $7.4 \times 10^{-6}$ & 2.03 & $8.5 \times 10^{-6}$ & $1.2 \times 10^{6}$ & 2.33 & $5.0 \times 10-4$ \\
\hline \hline
\end{tabular}

some earlier calculations. The results of Seckel, Stanev, and Gaisser [22] are in agreement both regarding normalization and shape, although they do not extend to as large energies as our calculation. The result of Moskalenko and Karakula [2] shows a significant difference in shape, related to their neglect of secondary interactions. By comparing our Figs. 1(a) and 1(b) with Fig. 1(c) it is obvious that secondary interactions and energy loss for muons cannot be neglected, even at relatively low energies. In fact by using the fluxes in Fig. 1(c) (where secondary interactions are unimportant) for the whole Sun, the result of Moskalenko and Karakula is roughly reproduced.

One can represent the neutrino fluxes by the simple parametrization

$$
\phi(E)= \begin{cases}N_{0} E^{-\gamma-1} /(1+A E), & E<E_{0}, \\ N_{0}^{\prime} E^{-\gamma^{\prime}-1} /(1+A E), & E>E_{0} .\end{cases}
$$

Although the form is the same as in Eq. (19) of [1] for the atmospheric fluxes, the physical interpretation is not as simple here due to the integration over impact parameter and the inclusion of the attenuation factor. Still, this form gives a good fit to the total attenuated fluxes in Fig. 4 resulting in the parameter values in Table II. ( $N_{0}^{\prime}$ is not fitted but given by the continuity condition at $E_{0}$.)

Neutrino telescopes measure neutrino fluxes indirectly through the Çerenkov light emitted from the muons and electrons produced in charge current neutrino interactions. The rate is, therefore, not directly proportional to the flux, but rather to the flux folded with the probability that the neutrino

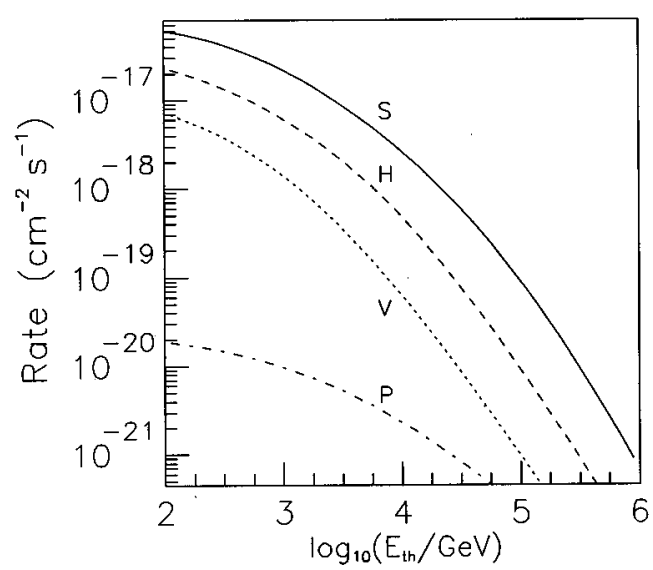

FIG. 5. The integrated rate of muon neutrino events in a neutrino telescope, as given by Eq. (16). The event rate for neutrinos from the Sun $(S)$ as compared to the ones based on the horizontal $(H)$, vertical $(V)$, and prompt $(P)$ Earth atmospheric fluxes. undergoes a charge current interaction and that the produced charged lepton reaches the detector, i.e., proportional to its range. Thus, the rate in a water or ice Çerenkov telescope is given by the quantity

$$
R=\int_{E_{\mathrm{th}}}^{\infty} d E \phi(E) \sigma^{\nu \rightarrow \ell}(E) \frac{\rho}{m_{p}} L(E)
$$

Here, $\rho$ is the density of the medium $\left(\rho \approx 1 \mathrm{~g} / \mathrm{cm}^{3}\right.$ for water/ice), $m_{p}$ is the proton mass such that $\rho / m_{p}$ is the target number density, and $L(E)$ is the range of the lepton. The lepton energy is not the same as the neutrino energy, but for the energies in this study it is a sufficiently good approximation. The muon range is given by

$$
L\left(E_{\mu}\right)=\frac{1}{\beta \rho} \ln \left(\frac{E_{\mu}+\alpha / \beta}{\alpha / \beta}\right)
$$

with $\alpha, \beta$ from the muon energy loss formula Eq. (9). The results of a numerical evaluation of the rate, Eq. (16), are shown in Fig. 5.

The rate that this flux would induce in neutrino telescopes under construction is very low. For example, the version of AMANDA currently being deployed will have an effective area of about $3 \times 10^{8} \mathrm{~cm}^{2}$ and a neutrino energy threshold of about $100 \mathrm{GeV}$. This would give one event per 2 years of running.

\section{NEUTRINO OSCILLATIONS}

If neutrinos are not massless there may be oscillations between the different weak eigenstates $\nu_{e}, \nu_{\mu}, \nu_{\tau}$. This phenomenon has been used to explain deficits of low energy solar neutrinos and atmospheric neutrinos in the GeV energy range. A number of particle physics experiments at accelerators and nuclear reactors have searched for oscillations. For a recent review of the field, see [23].

If neutrino oscillations indeed occur, they will affect also the high energy neutrino fluxes from the Sun. The situation is, however, very different from the case of low energy solar neutrinos, where the Mikheyev-Smirnov-Wolfenstein (MSW) effect [24] is the dominating source of oscillations [25]. The origin of the MSW effect is the different "index of refraction" for muon and electron neutrinos in matter and it only occurs when certain conditions for neutrino energy and nuclear number density are satisfied. These conditions are not fulfilled for the high energy neutrinos considered here and, since the fluxes are strongly attenuated when passing through the Sun (Fig. 3), the effect can be neglected. The oscillations could instead be of importance due to the large distance from the Sun as source to the detector at the Earth.

In our analysis of this, we assume that there are two mas- 

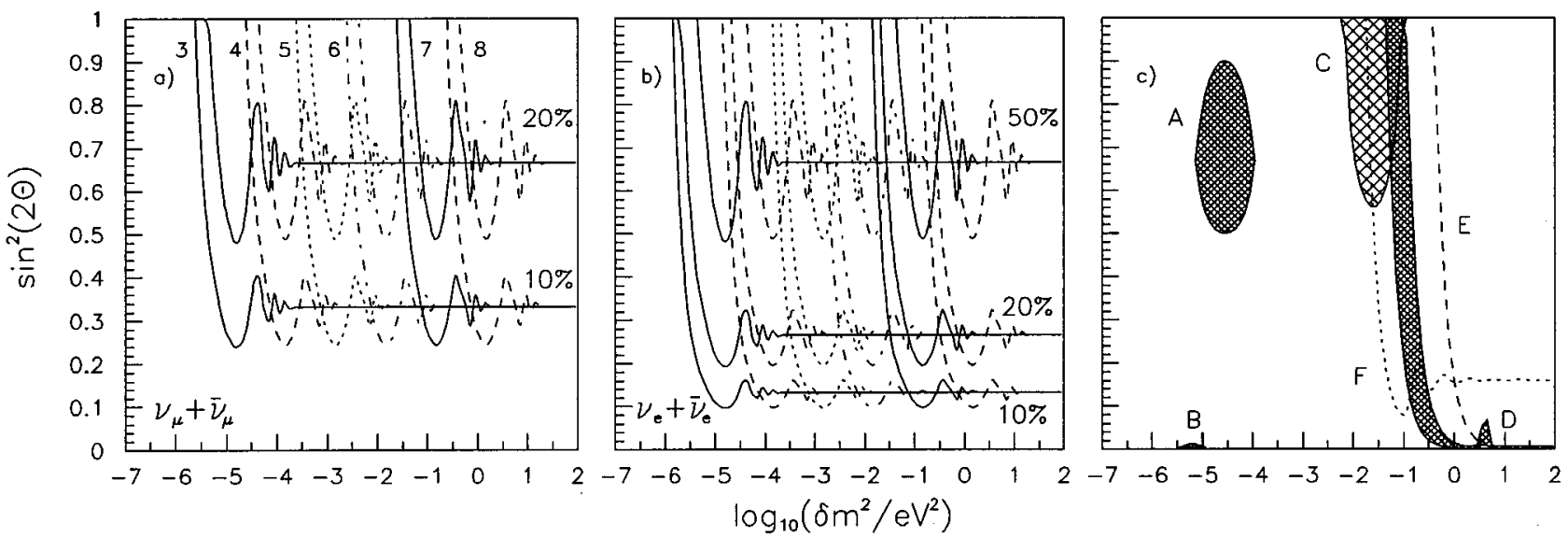

FIG. 6. Isolines for the indicated percentage (a) decrease of the muon neutrino flux and (b) increase of the electron neutrino flux due to $\nu_{\mu}-\nu_{e}$ oscillations as functions of the neutrino mixing angle $\theta$ and mass difference $\delta m^{2}=\left|m_{1}^{2}-m_{2}^{2}\right|$. Results, see Eq. (20), averages over energy bins in $\log _{10}\left\{E_{\nu}\right\}$ centered at $E_{\nu}=10^{n} \mathrm{GeV}$ with the indicated $n=3,4, \ldots, 8$. (c) Limits and not excluded regions based on different experiments, see text.

sive neutrino states that mix. The probabilities that a neutrino emitted as flavor $\ell$ is of flavor $\ell$ or $\ell^{\prime}$ at detection are given by

$$
\begin{gathered}
P_{\nu / \nu \ell}\left(E_{\nu}, x\right)=1-\sin ^{2}(2 \theta) \sin ^{2}\left(\frac{\delta m^{2} x}{4 E_{\nu}}\right), \\
P_{\nu / \ell^{\prime}}\left(E_{\nu}, x\right)=\sin ^{2}(2 \theta) \sin ^{2}\left(\frac{\delta m^{2} x}{4 E_{\nu}}\right),
\end{gathered}
$$

where $\theta$ is the neutrino mixing angle, $\delta m^{2}=\left|m_{1}^{2}-m_{2}^{2}\right|$ is the difference of the squared neutrino masses and $x$ is the traversed distance, i.e., $x=D$ for the Sun-Earth distance.

Figure 6 illustrates the effect of the neutrino oscillation as a function of these two basic oscillation parameters by showing isolines of the indicated percent changes of the neutrino fluxes, i.e., in the ratio

$$
\left\{\Phi_{\nu_{\mu}}\left(E_{\nu}\right) P_{\nu_{\mu} \nu_{\mu}}\left(E_{\nu}, D\right)+\Phi_{\nu_{e}}\left(E_{\nu}\right) P_{\nu_{e} \nu_{\mu}}\left(E_{\nu}, D\right)\right\} / \Phi_{\nu_{\mu}}\left(E_{\nu}\right)
$$

for the muon neutrino and similarly for the electron neutrino. The results are averages over energy bins in $\log _{10}\left(E_{\nu}\right)$ centered at $E_{\nu}=10^{n} \mathrm{GeV}$ with $n=3,4, \ldots, 8$ as indicated. Given the starting point with a larger muon neutrino flux than the electron neutrino flux, see Fig. 4, we show the $10 \%$ and $20 \%$ decrease of the muon neutrino flux and the $10 \%, 20 \%$, and $50 \%$ increase of the electron neutrino flux in Figs. 6(a) and 6(b), respectively.

Two main features can be observed here. First, the lower limit of $\delta m^{2}$ increases with increasing energy. Second, all curves coincide at the highest $\delta m^{2}$, where the oscillation length becomes much shorter than the Sun-Earth distance such that $\sin ^{2}\left(\delta m^{2} D / 4 E_{\nu}\right)$ will be averaged out to $1 / 2$.

Figure 6(c) illustrates experimental limits and acceptable regions. Regions $A$ and $B$ are, together with a very small region around $\sin ^{2} 2 \theta \sim 1$ and $\delta m^{2} \sim 5 \times 10^{-8} \mathrm{eV}^{2}$, the regions which could explain the solar neutrino problem [25]. The region marked $C$ is allowed based on observation of atmospheric neutrino fluxes [26]. $D$ is the region not excluded by the LSND experiment [27]. The limit $E$ is set by the Goesgen reactor experiment [28] and $F$ by the accelerator experiment LAMPF E734 [29]. Since these allowed regions are not overlapping, one can interpret (neglecting experimental uncertainties) that as either some result is incorrect or there are more neutrino species involved in the oscillation.

Given the low absolute flux of high energy solar neutrinos, the event rate in currently planned neutrino telescopes will be too low for revealing searches for, or studies of, neutrino oscillations.

\section{CONCLUSIONS}

We have calculated the high energy muon and electron neutrino fluxes arising from the interactions of cosmic ray particles with the solar matter. Our resulting muon neutrino flux agrees with that obtained by Seckel et al. [22], but our result extends a few orders of magnitude higher in energy. The muon neutrino flux in [2] is in disagreement with both these results, due to an oversimplified model where secondary interactions in the Sun are not taken into account.

These solar neutrino fluxes are 1 to 2 orders of magnitude larger than those from cosmic ray interactions in Earth's atmosphere, when integrated over the solid angle of the Sun as seen from the Earth. This opens a possibility to use the solar neutrino flux as a "standard candle" for neutrino telescopes. However, here one must also consider the angular spread introduced by the charged current interaction producing the detectable muon relative to the incoming neutrino direction. This deflection is typically $\sim 10^{\circ} \sqrt{10 \mathrm{GeV} / E_{\mu}}$. In addition, the experimental measurement of the muon direction also has a limited resolution. Taking these two effects together, the solar disc will typically cover less than $\sim 10 \%$ of the solid angle that has to be integrated over. The solar muon neutrino flux, which stays the same, would then have to be compared with a factor 10 , or more, increased atmospheric flux such that the two would be of the same order of magni- 
tude. Under these conditions, one would only have a factor 2 increase towards the Sun and the use of the Sun as a "standard neutrino candle" does not look so promising. Here one should also be aware of the very low absolute rate of events in a neutrino telescope of the size now under consideration. For example, we estimate the rate of neutrino events with $E_{\nu}>100 \mathrm{GeV}$ to be one per year in a detector covering $6 \times 10^{4} \mathrm{~m}^{2}$.

We have also investigated the potential for observing neutrino oscillations during the passage from the source in the Sun and a detector at the Earth. In principle, one could access an interesting region in the parameter plane of $\sin ^{2} 2 \theta$ and $\delta m^{2}$, but with the very low absolute rates it is beyond present neutrino telescopes.

The positive consequence of these small solar neutrino fluxes is that they will cause less of a background problem in attempt to detect neutrinos from other sources. Of particular interest here is the search for neutrinos from neutralino annihilation in the Sun, where the predicted rate can be up to an order of magnitude larger depending on the supersymmetric parameters [30]. A clear observation of this phenomenon would both demonstrate supersymmetry, i.e., physics beyond the standard model in particle physics, and the presence of nonbaryonic dark matter in the Universe.
[1] M. Thunman, G. Ingelman, and P. Gondolo, Astropart. Phys. (to be published).

[2] I. V. Moskalenko and S. Karakula, J. Phys. G 19, 1399 (1993).

[3] M. Kamionkowski, Phys. Rev. D 44, 3021 (1991).

[4] H. E. Haber and G. L. Kane, Phys. Rep. 117, 75 (1985).

[5] Amanda Collaboration, F. Pylav et al., in Cosmic Ray Conferences, Proceedings of the 23rd International Cosmic Ray Conference, Calgary, Canada, 1993, edited by R. B. Hicks et al. (World Scientific, Singapore, 1994), Vol. 4, p. 561.

[6] G. V. Domogatsky, in TAUP 93, Proceedings of the Third International Conference on Theoretical and Phenomenological Aspects of Underground Physics, Assergi, Italy, edited by C. Arpesella, E. Bellotti, and A. Bottino [Nucl. Phys. B (Proc. Suppl.) 35, 290 (1994)].

[7] J. G. Learned, in Neutrino 92, Proceedings of the XVth International Conference on Neutrino Physics and Astrophysics, Granada, Spain, edited by A. Morales [Nucl. Phys. B (Proc. Suppl.) 31, 456 (1993)].

[8] L. K. Resvanis, in TAUP 93 [6], p. 294.

[9] B. Andersson, G. Gustafson, G. Ingelman, and T. Sjöstrand, Phys. Rep. 97, 33 (1983).

[10] T. Sjöstrand, PYTHIA 5.7 \& JETSET 7.4, Comput. Phys. Commun. 82, 74 (1994).

[11] T. K. Gaisser, Cosmic Rays and Particle Physics (Cambridge University Press, Cambridge, England, 1990).

[12] P. Lipari, Astropart. Phys. 1, 195 (1993).

[13] P. Pal and D. P. Bhattacharyya, Nuovo Cimento C 15, 401 (1992).

[14] T. H. Burnett et al., in Proceedings of the XXI International Cosmic Ray Conference, Adelaide, Australia, 1990, edited by R. Protheroe (Graphic Services, Northfield, South Australia, 1990), Vol. 3, p. 101.
[15] S. Ahlen et al., Phys. Rev. D 46, 895 (1992).

[16] M. Honda, T. Kajita, K. Kasahara, and S. Midorikawa, Phys. Rev. D 52, 4985 (1995).

[17] A. Gregory and R. W. Clay, in CRC Handbook of Chemistry and Physics (CRC Press Inc., Boca Raton, Florida, 1982), p. F-175.

[18] J. E. Vernazza, E. H. Avrett, and R. Loeser, Astrophys. J. Suppl. 45, 635 (1981).

[19] J. Christensen-Dalsgaard, Stellar Structure and Evolution, Lecture Notes 1993 (Institut for Fysik og Astronomi, Aarhus Universitet, 1993), p. 147.

[20] Particle Data Group, L. Montanet et al., Phys. Rev. D 50, 1173 (1994).

[21] L. V. Volkova, Yad. Fiz. 31, 1510 (1980) [Sov. J. Nucl. Phys. 31, 784 (1980)].

[22] D. Seckel, T. Stanev, and T. K. Gaisser, Astrophys. J. 382, 652 (1991).

[23] L. Camilleri, Cern Report No. CERN-PPE/94-87 (unpublished).

[24] L. Wolfenstein, Phys. Rev. D 17, 2369 (1978); ibid. 20, 2364 (1979); S. P. Mikheyev and A. Yu. Smirnov, Yad. Fiz. 42, 1441 (1985); Sov. J. Nucl. Phys. 42, 913 (1985); Nuovo Cimento C 9, 17 (1986).

[25] N. Hata and P. Langacker, Phys. Rev. D 52, 420 (1995), and references therein.

[26] Y. Fukuda et al., Phys. Lett. B 335, 237 (1994).

[27] LSND Collaboration, C. Athanassopoulos et al., Phys. Rev. Lett. 75, 2650 (1995).

[28] V. Zacek et al., Phys. Lett. 164B, 193 (1985).

[29] L. A. Ahrens et al., Phys. Rev. D 31, 2732 (1985).

[30] J. Edsjö (private communication). 